University of Wollongong

Research Online

Faculty of Engineering and Information

Faculty of Engineering and Information

Sciences - Papers: Part A

Sciences

$1-1-2014$

Isolating plasma from blood using a dielectrophoresis-active hydrophoretic device

Sheng Yan

University of Wollongong, sy034@uowmail.edu.au

Jun Zhang

University of Wollongong, jz218@uowmail.edu.au

Gursel Alici

University of Wollongong, gursel@uow.edu.au

Haiping Du

University of Wollongong, hdu@uow.edu.au

Yonggang Zhu

Csiro Materials Science And Engineering

See next page for additional authors

Follow this and additional works at: https://ro.uow.edu.au/eispapers

Part of the Engineering Commons, and the Science and Technology Studies Commons

Research Online is the open access institutional repository for the University of Wollongong. For further information contact the UOW Library: research-pubs@uow.edu.au 


\title{
Isolating plasma from blood using a dielectrophoresis-active hydrophoretic device
}

\begin{abstract}
Plasma is a complex substance that contains proteins and circulating nucleic acids and viruses that can be utilised for clinical diagnostics, albeit a precise analysis depends on the plasma being totally free of cells. We proposed the use of a dielectrophoresis (DEP)-active hydrophoretic method to isolate plasma from blood in a high-throughput manner. This microfluidic device consists of anisotropic microstructures embedded on the top of the channel which generate lateral pressure gradients while interdigitised electrodes lay on the bottom of the channel which can push particles or cells into a higher level using a negative DEP force. Large and small particles or cells $(3 \mu \mathrm{m}$ and $10 \mu \mathrm{m}$ particles, and red blood cells, white blood cells, and platelets) can be focused at the same time in our DEP-active hydrophoretic device at an appropriate flow rate and applied voltage. Based on this principle, all the blood cells were filtrated from whole blood and then the plasma was extracted with a purity of $94.2 \%$ and a yield of $16.5 \%$ at a flow rate of $10 \mu \mathrm{L}$ min-1. This solved the challenging problem caused by the relatively low throughput of the DEP based device. Our DEP-active hydrophoretic device is a flexible and tunable system that can control the lateral positions of particles by modulating the external voltages without redesigning and fabricating a new channel, and because it is easy to operate, it is easily compatible with other microfluidic platforms that are used for further detection.
\end{abstract}

\section{Keywords}

device, isolating, blood, hydrophoretic, plasma, active, dielectrophoresis

\section{Disciplines}

Engineering | Science and Technology Studies

\section{Publication Details}

Yan, S., Zhang, J., Alici, G., Du, H., Zhu, Y. \& Li, W. (2014). Isolating plasma from blood using a dielectrophoresis-active hydrophoretic device. Lab on a Chip: miniaturisation for chemistry, physics, biology, materials science and bioengineering, 14 (16), 2993-3003.

\section{Authors}

Sheng Yan, Jun Zhang, Gursel Alici, Haiping Du, Yonggang Zhu, and Weihua Li 


\title{
Isolating plasma from blood using a dielectrophoresis-active hydrophoretic device
}

\author{
Sheng Yan, ${ }^{a}$ Jun Zhang, ${ }^{a}$ Gursel Alici, ${ }^{a}$ and Haiping $\mathrm{Du},{ }^{b}$ Yonggang $\mathrm{Zhu}^{* c, d}$ and Weihua Li ${ }^{* a}$ \\ Received (in $X X X, X X X)$ Xth $X X X X X X X X X 20 X X$, Accepted $X t h X X X X X X X X X 20 X X$ \\ DOI: $10.1039 / \mathrm{b} 000000 \mathrm{x}$
}

Plasma is a complex substance that contains proteins and circulating nucleic acids and viruses that can be utilised for clinical diagnostics, albeit a precise analysis depends on the plasma being totally free of cells. We proposed using a dielectrophoresis (DEP)-active hydrophoretic method to isolate plasma from blood in a high-throughput manner. This microfluidic device consists of anisotropic microstructures embedded on the top of the channel which generate lateral pressure gradients while interdigitised electrodes lay on the bottom of the channel which can push particles or cells into a higher level using negative DEP force. Large and small particles or cells ( $3 \mu \mathrm{m}$ and $10 \mu \mathrm{m}$ particles, and red blood cells, white blood cells, and platelets) can focus at the same time in our DEP-active hydrophoretic device under an appropriate flow rate and applied voltage. Based on this principle, all the blood cells were filtrated from whole blood and then plasma was extracted with a purity of $94.2 \%$ and a yield of $16.5 \%$ at a flow rate of $10 \mu \mathrm{L} \mathrm{min}{ }^{-1}$. This solved the challenging problem caused by the relatively low throughput of the DEP based device. Our DEP-active hydrophoretic device is a flexible and tunable system that can control the lateral positions of particles by modulating the external voltages without redesigning and fabricating a new channel, and because it is easy to operate, it is easily compatible with other microfluidic platforms that are used for further detection.

\section{Introduction}

Human blood including leukocytes or white blood cells (WBCs), erythrocytes or red blood cells, and platelets and plasma ${ }^{1}$. WBCs play a significant role in the immune system, account for $1 \%$ of whole blood cells, and range from 7 to $30 \mu \mathrm{m}$ in size. Red blood cells have a discoid shape and are 7 to $8 \mu \mathrm{m}$ in size; they are the most common cells, accounting for $98 \%$ of all blood cells ${ }^{2}$. Platelets are 1 to $3 \mu \mathrm{m}$ in size and are responsible for formatting blood clots ${ }^{3}$. Plasma is a straw coloured aqueous medium free of cells that acts as a host to a myriad of analytes containing metabolites, proteins, circulating nucleic acids (CNAs), and other organisms. An accurate analysis depends on the plasma being totally free of cells, and therefore the contaminants which might otherwise influence the results of any detection must be removed 2 .

Two conventional mechanisms - centrifugation and filtration remain the most common methods of separating blood plasma in the laboratories ${ }^{4}$. While these methods are simple, the procedures are generally labour intensive, time consuming ${ }^{2}$, and sometimes can impair the collection of analytes of interest (e.g. high speed centrifugation with $>3000 \mathrm{~g}$ ). The recent advance in microfabrication and microfluidics technologies has provided impetus for developing microfluidic techniques for blood plasma separation. Microfluidic techniques have many advantages over conventional methods such as use of small volume of blood samples (e.g., microlitres instead of millilitres), short analysis time (e.g., seconds to minutes), user-friendliness and capability of automation. Use of microfluidics can lower the analysis cost significantly and miniaturise the analysis device, making it attracting for portable and low cost analysis of biological samples.

Microfluidic techniques are usually categorised into an active and passive isolation of plasma from whole blood. Passive separation methods can sort particles by their physical characteristics such as size, shape, and density, using only the shapes of the micro-channels or micro-structures embedded in the micro-channels, the flow, and the hydrodynamic forces. Sedimentation is the oldest method and has been adapted to micro-scale systems for collecting plasma ${ }^{5}$. Although undiluted whole blood can be easily operated in microfluidic sedimentation devices, the separation throughput is relatively low because any increase in the flow rate will cause inefficient separation. Hence, each experiment will last several hours. Microfiltration is another example of taking the macro-scale principle into the micro-scale world for isolating plasma. Membranes and pores have been designed to prevent blood cells from passing through so the plasma can be extracted from blood ${ }^{6-8}$. However, the major bottleneck with these methodologies is the problem of clogging, which affects their performance after the filter becomes saturated. Thus, these techniques can only operate under a given volume and with a diluted sample before the filters become saturated. The deterministic lateral displacement (DLD) was first proposed and 
verified by Huang et al. ${ }^{9}$ with micro-pillars within a microchannel. This method was successfully applied to separate blood cells and isolate plasma ${ }^{1}$. It still suffers from various drawbacks such as the need for an extremely low flow rate, a precise sheath flow, and the high cost of fabrication. Meanwhile, the narrow gaps between the pillars embedded in the micro-channel bring the risk of clogging. Hydrodynamic separation techniques that depend on viscous forces are generally used to separate the plasma from blood. Yang et al. ${ }^{10}$ designed a novel microfluidic device that used the Zweifach-Fung law where $100 \%$ purity (image analysis) was achieved with $25 \%$ plasma yield and an extraction rate of $4 \mu \mathrm{L} \mathrm{min}{ }^{-1}$. In the microchannels, the Reynolds number ( $R e=U d / v$, where $U$ is the flow velocity, $d$ is the channel width and $v$ the kinematic viscosity of the fluids) is very low (i.e., $R e<=1$ ), the viscous force dominates and the flow is laminar. Therefore, hydrodynamic separation operates at low $R e$ in most blood extraction processes. Di Carlo ${ }^{11}$ and our group ${ }^{12}$ demonstrated that the inertial lift forces can actually focus and separate microparticles and cells at higher Reynolds numbers. Di Carlo et al. ${ }^{13}$ introduced a device using such a principle to separate plasma and bacteria from blood cells. Although a high flow rate was achieved in an inertial microfluidic device, the small cells (E.Coli) cannot be focused well and were not entirely separated from the blood. The plasma was not free of cells, which will influence the results of further analysis. Recently, Choi et al. proposed a novel approach to sort and focus micro-particles in micro-channels with slanted grooves, called hydrophoresis ${ }^{14-19}$. Hydrophoresis is a sheathless and passive technique that utilises anisotropic micro-structures to induce a pressure gradient ${ }^{18}$. The concept of hydrophoresis was verified by purifying platelets from blood using a fast method for low stress and high throughput ${ }^{3}$. A hydrophoretic filtration device was also used to isolate the white blood cells (WBCs) from red blood cells ${ }^{20}$. Although hydrophoresis is a powerful and robust sorting mechanism, it still has some limitations. For instance, according to hydrophoretic ordering that has been studied experimentally, only particles with diameters normally larger than half of channel height can be ordered and focused ${ }^{14}$. For separating platelets with a diameter of 1-3 $\mu \mathrm{m}$ in a hydrophoretic device, the maximum height of any gaps should be less than $6 \mu \mathrm{m}$, which prevents the passage of WBCs and red blood cells through the micro-channel. Therefore, plasma cannot be effectively isolated in hydrophoretic devices. In the hydrophoretic filtration experiment, the WBCs $(D>8 \mu \mathrm{m})$ could not flow through the channel easily that was $7.8 \mu \mathrm{m}$ high and with an obstacle height of $4 \mu \mathrm{m}$, which might lead to clogging. Moreover, high throughput is difficult to achieve in such narrow channels. Even though parallelisation strategy can be used to increase the throughput, it is impossible to fabricate channels with different sizes which are required for different biological samples.

Active techniques using external force fields such as acoustic ${ }^{21}$, magnetic ${ }^{22}$ and dielectric ${ }^{23-34}$ can also be used to sort particles or cells. Acoustic forces induced by high frequency resonators rely on their size, density, and compressibility ${ }^{35}$. Even though the theory of magnetically isolating plasma from whole blood exists, it is impractical because of the need for additives to supply paramagnetic red blood cells ${ }^{2}$. As for isolating plasma electrically, little work has been reported so far. Nakashima et al.
${ }^{36}$ proposed a system of combining capillary and dielectrophoretic forces for isolating plasma where the cells were attracted to weak electric fields under negative DEP forces while the plasma was collected by capillary forces. However, the plasma yield was very low $(6 \%)$ under a voltage of $10 \mathrm{~V}$ at $1 \mathrm{MHz}$. No external mechanical sources such as centrifugal machines and syringe pumps were used in this experiment at the expense of time. Unlike their passive counterparts, active methods have a low flow rate and required specialised buffers. To the best of the authors' knowledge, the use of hydrophoretic devices to extract plasma has not been reported and very few DEP devices related to plasma isolation at high flow rate have been presented. The flow rate of hydrophoretic devices is relatively high compared to that of DEP devices. The DEP force can be tuned by varying the external voltages to manipulate particles, and has the potential for setting up a tunable system. Combining DEP with hydrophoresis thus benefits the best from the two methods. In a previous work, the concept of "DEP-active hydrophoretic focusing" was proposed and it was shown that the focusing patterns of particles ( $>8 \mu \mathrm{m}$ in diameter) could be modulated by varying the voltage at a high throughput ${ }^{37}$. However, the separation of both small and large particles has not been demonstrated.

The aim of this paper is to report the new development of the device that can separate both large and small particles with high throughput. Such a development can find applications in a range of applications such as sensor devices, pathology test and so on. The brief outline of the paper is as follows. Section II will introduce experimental details including methodology, device design, materials and experimental setup. Numerical simulation will be introduced in Section III. The main results will be presented in Section IV which include both numerical and experimental data and an application example of blood plasma separation. The main conclusion will be drawn in Section V.

\section{Experimental details}

\section{A. Methodology}

To separate both large and small particles, a new design is proposed with optimized flow and DEP conditions. The flow velocity is reduced to allow for the separation of smaller particles. A higher DEP voltage will be used in order to generate larger DEP force to push smaller particles high enough in the channel. To increase the throughput, a larger channel cross section is used.

The mechanism of hydrophoresis has been studied experimentally ${ }^{3}$. The critical diameter to be focused in the channel is defined as half the height of the channel. Normally, particles travel back and forth along the transverse direction following the rotational flow. However, particle-groove interaction diffuses the large particles whose diameters exceed the critical diameter out of their streamlines and lead to equivalent flow paths; this is called hydrophoretic ordering. In our experiments, the particles exerting a negative DEP force will be levitated in the suspending medium. The DEP force also depends on the applied field and the diameter of particles, respectively, which implies larger particles or cells have a higher levitation position and a higher voltage also tends to push particles or cells to a higher level in the channel. Therefore, 
interdigitated electrodes were patterned onto the bottom of the channel so that particles exerting a negative DEP force were pushed into a narrow space. As a result, the particles have more opportunity to interact with the grooves and induce hydrophoretic ordering by steric hindrance. Hence, large and small particles or cells $(3 \mu \mathrm{m}$ and $10 \mu \mathrm{m}$ particles, and Red blood cells, WBCs, and platelets) can focus simultaneously in our DEP-active hydrophoretic device under the appropriate flow rate and applied voltage even though their diameters are less than the critical diameter, which enables all the blood cells to separate from the blood and obtain plasma with high purity.

Two important factors were used to evaluate how well the blood plasma separated with regards to its purity and yield. The purity of plasma is defined as: $1-c_{p} / c_{f}$, where $c_{p}$ is the number of red blood cells per milliliter in the collection outlet and $c_{f}$ is the number of red blood cells in the inlet fraction. The yield of plasma is the percentage of the volume of collected plasma from the collection outlet over the total volume of blood. Our DEPactive hydrophoretic device is a flexible and tunable system that can focus the lateral positions of particles by changing the external voltages without redesigning and fabricating a new channel.

\section{B. Design and fabrication of a DEP-active hydrophoretic device}

Fig. 1 shows a schematic drawing of the microfluidic device. The width of the channel is the key factor that impacts on the focusing positions of different particles. When the channel is wider than $400 \mu \mathrm{m}$, particles with different sizes are focused onto the sidewall of the channel with a small deviation known as the focusing mode ${ }^{18}$. A $500 \mu \mathrm{m}$ wide microfluidic channel is designed so that all the blood cells with distinguishing diameters can work in a focusing mode and will have slightly different focusing positions. The WBCs, red blood cells, and platelets migrating along the sides of the micro-channel are collected from the waste outlet, while the free-cell plasma is extracted from the collection outlet.

Those particles with diameters $D$ that are larger than half of the height of the main channel $H$ assumed hydrophoretic ordering ${ }^{38}$. The steric interaction between grooves and beads deflects particles from rotational flow and leads a new equivalent flow path. This anisotropic structure generates a pressure gradient from the sides of channel which induces a rotational flow consisting of upward, focusing, downward, and deviation flow (Fig. 2) ${ }^{15}$. Such convective vortices drive cells that move in the cross section and follow the rotational flows. When the large cells with diameters larger than half the height of the channel move to the sides of the channel, the steric effect induces the cells to diffuse out of convective vortices and remain near the sidewalls (Fig. 2a). When the small cells reach the sides of the channel they go downwards and deviate from the sides by focusing flow. In short, the steric hindrance for small cells is negligible so they can migrate back and forth in the micro-channel without focusing.

The DEP-active hydrophoretic device was designed to improve the throughput of this device. The micro-channel height for the two sections are $H_{t}=90 \mu \mathrm{m}$ and $H=45 \mu \mathrm{m}$, respectively. The blood cells could not form hydrophoretic ordering in our experiments because the largest blood cells were approximately $10 \mu \mathrm{m}$ in diameter. Therefore, micro-electrodes were used to generate the non-uniform electric field where the negative DEP force would levitate the cells into a higher position in the $z$-direction and enhance the cell-groove interaction and generate hydrophoretic ordering. The higher position the cells have, the easier they form hydrophoretic ordering, and therefore the small cells (platelets) could also be focused in the micro-channel after applying a strong electric field (Fig. 1). The channel $W$ was $500 \mu \mathrm{m}$ wide and $H_{t}=90 \mu \mathrm{m}$ high, the main channel was $H=45 \mu \mathrm{m}$ high and its length was $L=10 \mathrm{~mm}$. The grooves were crescent shaped with a small curvature of $250 \mu \mathrm{m}$ and a large curvature of $300 \mu \mathrm{m}$ (Fig. $1 \mathrm{~b}$ and Fig. 2).

The double-layer master mould was fabricated on a silicon wafer using two-step photolithographic techniques. The first layer of photolithography was defined as the main channel; the second one with the pattern of grooves was aligned to lie on the top of structures in the first layer. The first layer of photoresist (SU-8 2050, MicroChem Corp., Newton, MA) was spun on a clean silicon wafer by a three-step coating cycle (500 rpm for 20s, 2000 $\mathrm{rpm}$ for $20 \mathrm{~s}$, and $4000 \mathrm{rpm}$ for $40 \mathrm{~s}$ ). Then, the silicon wafer was baked at $65{ }^{\circ} \mathrm{C}$ for 2 minutes and $95{ }^{\circ} \mathrm{C}$ for 7 minutess. After exposed to UV light through the first photomask, another twostep hard bake $\left(65{ }^{\circ} \mathrm{C}\right.$ for 3 minutess and $95{ }^{\circ} \mathrm{C}$ for 7 minutess) was conducted. Afterwards, the SU-8 pattern was developed in SU-8 developer solution for 6 minutess and rinsed with isopropyl alcohol (IPA). In the second-step lithography, the photoresist was spin-coated on the previous mould, which was the same procedure with first-step photolithography. The second photomask was carefully aligned with the previously developed SU-8 patterns and then UV light went through the second photomask to expose the second layer of photoresis. After baking and developing, the double-layer mold was treated by trichlorosilane to deposit a mono-layer of silane on the surface. A PDMS mixture with 10/1 ratio of curing agent (Dow Corning, Midland, MI) was poured over the silicon master, degassed and baked at $65{ }^{\circ} \mathrm{C}$ for $2 \mathrm{~h}$. The devices were peeled off from the silicon master and inlet and outlet holes were punched with a custom needle tip. The PDMS was sealed with glass slides after exposure to oxygen plasma (PDc-002, Harrick Plasma, Ossining, $\mathrm{NY}$ ) for $3 \mathrm{~min}$. Other details of this fabrication can be found in our previous work ${ }^{39-40}$.

\section{Material preparation}

Red fluorescent microparticles with a diameter of $3 \mu \mathrm{m}$ and green fluorescent beads of diameter $10 \mu \mathrm{m}$ (Thermo Fisher Scientific., USA) were used in our experiments. They were suspended in deionized (DI) water, with Tween 20 (Sigma-Aldrich, Product No. P9416) added to this aqueous medium to impede the beads from sedimentation and aggregation. The human blood samples were extracted from healthy donor. The samples were diluted $100 \times(0.45 \% \mathrm{hct})$ in an isotonic sucrose buffer before separating the plasma.

\section{Experimental setup}

A sinusoidal signal with frequency $1 \mathrm{MHz}$ was generated by a waveform generator (33250A, Agilent, USA) and amplified by an RF power amplifier (TIA-1000-1R8-2, Mini-Circuits, USA). The applied frequency in all experiments was set to $1 \mathrm{MHz}$. The copper wires soldered onto the pads were connected to the power amplifier to activate the electrodes. A syringe pump (Legato 100, 
Kd Scientific) was used to inject the particle suspension into the micro-channel. The microfluidic device was placed under an inverted microscope (CKX41, Olympus, Japan) and the particle trajectories were captured by a CCD camera (Rolera Bolt, Qimaging, Australia). An image processing program, Q-Capture Pro 7 (Q-imaging, Australia), was used to measure the focusing positions and the widths of the beads. The concentration of particles or cells collected from different outlets was measured with a hemacytometer.

\section{Numerical simulation}

The particle trajectories and flow characteristics in the DEPactive hydrophoretic device were calculated using the finite element software (COMSOL Multi-physics 4.3 COMSOL, Burlington, MA). The geometry for simulation was identical to the experimental channel. The laminar flow module was utilised to solve the fluid flow in the three-dimensional model. No-slip boundary condition was set to the channel walls. The physical property of fluid was set to incompressible flow. The flow rate at the inlet was set to $15 \mu 1 \mathrm{~min}^{-1}$ and the pressure at the outlet was set to zero. The pressure and velocity vectors on the cross sections can be solved in the "Navier-Stokes Mode". The electric currents module was employed to calculate the non-uniform electric field generated by activated electrodes. $20 \mathrm{~V}_{\mathrm{p}-\mathrm{p}}$ AC signal with a frequency of $1 \mathrm{MHz}$ was applied to the electrodes. A frequency solver was used to compute the electric field. Finally, the particle tracing module can couple DEP force and drag force to simulate the particle trajectories. Also, the equation of DEP force and drag force was already embedded in the COMSOL without secondary development. Therefore, DEP force and drag force were automatically computed accord ing to the electric field and fluid field, respectively. At the inlet, particles were evenly released along the channel width. The particle density and diameter were set to $1050 \mathrm{~kg} \mathrm{~m}^{-3}$ and $3 \mu \mathrm{m}$, respectively. The time for beads migrating in the channel was set to $0.5 \mathrm{~s}$ and the interval time was set to $10^{-4} \mathrm{~s}$, which provided smooth particle trajectories. In the $z$-axis a DEP force and drag force were exerted onto particles in the DEP-active hydrophoretic device. The DEP force $F_{\text {DEP }}$ on spherical particles is given by ${ }^{41}$ :

$$
F_{\mathrm{DEP}}=2 \pi \varepsilon_{m} r^{3} \operatorname{Re}[K(\omega)] \nabla E_{\mathrm{RMS} z}^{2}
$$

where $\varepsilon_{m}$ is the absolute permittivity of the suspending medium, $r$ donates the particle radius, $\nabla E_{\mathrm{RMS} z}{ }^{2}$ is the gradient of the square of the applied field $E_{\mathrm{RMS}}$ in the $z$-axis, and $K(\omega)$ refers to the Clausius-Mossotti (CM) factor. Re indicates the real part of the factor is considered. $K(\omega)$ depends on the complex permittivities of the particle and the suspending medium, and the frequency of the external electric field, via,

$$
K(\omega)=\left(\varepsilon_{p}^{*}-\varepsilon_{m}^{*}\right) /\left(\varepsilon_{p}^{*}+2 \varepsilon_{m}^{*}\right)
$$

where $\varepsilon^{*}=\varepsilon-i \sigma / \omega\left(i^{2}=-1\right)$ is the complex permittivities, $\sigma$ is the electrical conductivity and $\omega$ is the frequency of the electric field. The subscripts $p$ and $m$ denotes the particle and suspending medium, respectively. The drag force at low Reynolds numbers can be expressed using Stokes law ${ }^{42}$ :

$F_{d r a g}=-6 \pi r \eta v_{m z}$ where $\eta$ refers to the viscosity of the medium, $v_{m z}$ is the velocity of the flow.

The DEP and drag forces can be modulated independently. $F_{\text {DEP }}$ changes with the applied voltage while $F_{d r a g}$ varies with the flow rate.

\section{Results and discussion}

\section{A. Modelling of particle trajectories}

Fig. 2a shows the simulated particle trajectories in the DEP-active hydrophoretic device. The flow rate applied along the $y$-axis was set to $15 \mu 1 \mathrm{~min}^{-1}$. When released evenly from inlet, particles are pushed by negative DEP induced by electric field to a high level in $z$-axis, where particles are mainly exposed to deviation flows. Thus, they will rapidly reach the sidewalls of channel following the deviation flow. Steric hindrance then occurs to prevent particles moving back and forth in $x$-axis and keep them along the sidewalls. After that, the particles are focused gradually.

Fig. $2 \mathrm{~b}$ illustrates the schematic diagram of the micro-channel, and the insets are the enlarged view of the simulated velocity vectors and pressure field in two cross-sections around the grooves. The pressure field changes significantly around the grooves. The maximum pressure appears near the sidewalls and the pressure is smallest near the centre of channel. The velocity vectors are identical with the pressure field, which drives the vortices consisting of upward, deviation, downward, focusing flows. Due to the anisotropic structure of the channel, the drag force in the cross-section varies periodically through the channel. The DEP forces, however, keep the constant value in the same height in the channel, which will push particles higher or lower to balance the drag forces. Therefore, the trajectories of particles are not ideally straight in the channel with grooves, instead of helical motion with small radius (the inset of Fig. 1a).

\section{B. Movements of $3 \mu \mathrm{m}$ particle in the DEP-active hydrophoretic channel}

Since the small beads are not easily focused in the microfluidic channel, the conditions for focusing $3 \mu \mathrm{m}$ beads are first studied. The effect of voltage and flow rate on the focusing performance of $3 \mu \mathrm{m}$ fluorescent beads are shown in Fig. 3. The $3 \mu \mathrm{m}$ microparticles were injected into the device and distributed themselves evenly at the inlet as the flow rate was changed from 5 to $20 \mu \mathrm{L}$ $\min ^{-1}$ and the voltages varied from 10 to $30 \mathrm{~V}_{\mathrm{p}-\mathrm{p}}$. Fig. 3a shows trajectories of particles along the whole channel, which agree reasonably well with the simulation result shown in Fig. 2a. Beads following the deviation flows are driven to the sidewalls of the channel. Steric effect occurs to remain the beads moving along the sidewalls. Finally, the particles are focused into lines at the outlet. Fig. $3 b$ shows the optical micrographs of particle trajectories of $3 \mu \mathrm{m}$ beads passing the DEP-active hydrophoretic device. The time interval of each image was $1 / 7 \mathrm{~s}$ and the images were superposed.

Fig. $3 \mathrm{c}$ shows the focusing positions with different flow rates. The focusing positions of the $3 \mu \mathrm{m}$ beads were symmetrical about the centre of the channel. As the flow rate is increased from 5 to $20 \mu \mathrm{L} \mathrm{min} \operatorname{mon}^{-1}$ at an applied voltage of $10 \mathrm{~V}_{\mathrm{p}-\mathrm{p}}$, the focusing positions of the $3 \mu \mathrm{m}$ beads deviated from the sidewalls, changing from 70.6 and $424.3 \mu \mathrm{m}$, to 88.4 and $406.4 \mu \mathrm{m}$. Since the higher lateral pressure gradient was induced by the high flow 
rate from the sides to the centre of the channel, the beads were exposed to a higher transverse flow which deflected them away from the sidewalls of channel. Therefore, the particles moved inwards as the flow rate was increased. Although similar behaviour was observed at voltages of 20 and $30 \mathrm{~V}_{\mathrm{p}-\mathrm{p}}$, the variation of focusing positions with flow rate is much less pronounced when compared to that at $10 \mathrm{~V}_{\mathrm{p}-\mathrm{p}}$. This variation is reduced even more at a higher applied DEP voltage. For example, at a DEP voltage of $30 \mathrm{~V}_{\mathrm{p}-\mathrm{p}}$, the particles migrated closer to the sidewalls and remained so along then whole channel. The small variation of focusing position along the channel can be regarded as a stable condition in our experiment. With the increase of voltages, the focusing positions of the particles allow the focusing width to be estimated (Fig. 3d). A the voltage of $10 \mathrm{~V}_{\mathrm{p}-\mathrm{p}}$ The focusing width increased significantly with the increasing flow rate in the range studied, i.e., from 5 to $20 \mu \mathrm{L} \mathrm{min}{ }^{-1}$. The beads were not levitated high enough to form hydrophoretic ordering because a low electric field was generated and a high flow rate was applied. The beads were not focused along the sidewalls of the channel any more. Instead, the beads were migrating back and forth from the sides to the centre of the channel. In contrast, at the applied voltage of 20 and $30 \mathrm{~V}_{\mathrm{p}-\mathrm{p}}$, the effect of flow rate on the focusing width is almost negligible. There is only a slight increase of the focusing width with the flow rate at $20 \mathrm{~V}_{\mathrm{p}-\mathrm{p}}$.

\section{Movements of $10 \mu \mathrm{m}$ particle in the DEP-active hydrophoretic channel}

In order to investigate the ability of the device for simultaneously separating beads with different sizes, the particle trajectories of $10 \mu \mathrm{m}$ beads were studied under the same conditions as $3 \mu \mathrm{m}$ beads (Fig. 4). The optical micrographs in Fig. 4a show that the particles assumed a hydrophoretic ordering and focused themselves onto the sides of the channel under all conditions studied, which differs from the observations of the $3 \mu \mathrm{m}$ beads. Since the DEP force on the particle is proportional to the cube of their diameter, the $10 \mu \mathrm{m}$ beads experiencing higher DEP forces are levitated to a higher equilibrium position in the $z$-direction, and the interaction between particle and groove was relatively intensive and the steric effect occurred easily. Therefore, $10 \mu \mathrm{m}$ beads can be focused quite well in the DEP-active hydrophoretic device under the same conditions applied to the $3 \mu \mathrm{m}$ beads.

Fig. $4 \mathrm{~b}$ and Fig. $4 \mathrm{c}$ show the focusing positions and widths of the $10 \mu \mathrm{m}$ beads at different flow rates, respectively. The focusing positions changed as the flow rate increased in a similar way as those observed for the $3 \mu \mathrm{m}$ beads. The beads were deflected from the sides of channel by a higher transverse flow as the flow rate increased, and the beads tended to move closer to the sidewalls as the voltages increased. Choi and Park ${ }^{16}$ had a similar result when they found that the lateral positions of the beads decreased when the height of the channel was lowered. Actually, applying a higher voltage can be the equivalent of lowering the height of the channel because the particles are pushed into a narrower space and can only migrate in the restricted area. Fig. $4 \mathrm{c}$ revealed that the focusing width of the 10 $\mu \mathrm{m}$ particles remained almost constant and was independent of the flow rate and voltage. The focusing width is very close to the diameter of the particles, indicating that their trajectories overlapped together and focused well. Because the particles were located in narrow ranges on the $z$-axis by the negative DEP forces induced by the interdigitated electrodes, they were exposed to the same flow pressure. This indicates they had a uniform lateral position and a minor change in their focusing width.

\section{DEP-active hydrophoretic filtration of micro-particles}

Filtration was first tested with $3 \mu \mathrm{m}$ diameter (red) and $10 \mu \mathrm{m}$ diameter (green) fluorescent particles whose sizes were comparable to the platelets, Red blood cells and WBCs. Fig. 5a shows the trajectories of the $3 \mu \mathrm{m}$ and $10 \mu \mathrm{m}$ particles captured at a flow rate of $15 \mu \mathrm{min}^{-1}$ and a voltage of $20 \mathrm{~V}_{\mathrm{p}-\mathrm{p}}$. The average linear velocity of particles flowing through the $500 \times 45 \mu \mathrm{m}$ channel is estimated to be $1.11 \mathrm{~cm} \mathrm{~s}^{-1}$. Similar patterns were obtained from different particles, except for the focusing positions. The $3 \mu \mathrm{m}$ and $10 \mu \mathrm{m}$ particles migrated along the sidewalls of the channel and were finally collected at the waste outlet. As a result, the fluid through the collection channel contains no or only a few micro-particles. The current experiment showed that, after filtration, $99.4 \%$ of $3 \mu \mathrm{m}$ beads and $99.8 \%$ of $10 \mu \mathrm{m}$ beads were removed from initial particle-laden fluid solution (Fig. 5b). Although the $3 \mu \mathrm{m}$ and $10 \mu \mathrm{m}$ beads did not satisfy the hydrophoretic ordering, focusing patterns were still observed under an external applied voltage, which generated negative DEP and pushed the particles into a narrow space in the $z$-axis. The large particles ( $10 \mu \mathrm{m}$ diameter) and small particles ( 3 $\mu \mathrm{m}$ diameter) were focused simultaneously in the DEP-active hydrophoretic channel, which enabled small cells (platelets) and large cells (red blood cells and WBCs) to focus in our microfluidic device. Therefore, plasma can be extracted from the collection outlet.

Particles migrating through the micro-channels also experienced inertial lift forces such as the shear gradient and wall-induced lift forces under a high flow rate. The particle Reynolds number $R_{p}$ is the ratio of particle inertia to the viscous force ${ }^{14}$, i.e. $\mathrm{R}_{\mathrm{p}}=\left(\rho D^{2} U\right) /\left(\mu D_{h}\right)$, where $\rho$ represents the fluid density, $D$ is the particle diameter, $\mu$ is the dynamic fluid viscosity and $D_{h}$ is the hydraulic diameter defined as $D_{h}=$ $2 W H /(W+H)$. For $\mathrm{R}_{\mathrm{p}}>1$, particle inertia was dominant and the equivalent paths of particles under hydrophoretic ordering were affected by inertial lift forces. The particle Reynolds numbers were 0.02 and 0.0018 for $10 \mu \mathrm{m}$ and $3 \mu \mathrm{m}$ beads at a flow rate of $15 \mu 1 \mathrm{~min}^{-1}$, respectively. Therefore, the particle inertial effect can be neglected in our experiments.

\section{E. Isolating plasma from diluted whole blood}

The DEP-active hydrophoretic method was also used for isolating plasma from diluted whole blood. The flow rate applied for isolating plasma was $10 \mu 1 \mathrm{~min}^{-1}$ and the external voltage was 20 $\mathrm{V}_{\mathrm{p}-\mathrm{p}}$. Choi et.al ${ }^{15}$ found when the hematocrit of blood was higher than $1 \%$, cell-cell interaction wouldn't be negligible. That is, blood cells will flow adjacent to each other and their cell-cell interaction may influence the focusing result of blood cells. Hence, the whole blood cells should be diluted to avoid the cellcell interaction. Therefore, diluted blood $(0.45 \%$ hct $)$ is prepared to improve the focusing performance of blood cells. The optical micrographs in Fig. 6a show the focusing patterns of blood cells. Those blood cells which formed hydrophoretic ordering then focused into the sidewalls of channel and were then collected from the waste outlet, while plasma was being extracted from the collection outlet. The blood cells had similar trajectories to the 
fluorescent particles, which show how effectively the biological cells were isolated in the DEP-active hydrophoretic channel. Although Red blood cells have a discoid shape and a diameter of 6.2-7.9 $\mu \mathrm{m}$ and a thickness of 1.7-2.6 $\mu \mathrm{m}^{20}$, they formed hydrophoretic ordering and migrated along the sides of the channel because they had a hydrodynamic diameter of $7.7 \mu \mathrm{m}^{43}$. When the diluted blood $(0.45 \%$ hct $)$ was injected into the channel, the blood cells were still focused onto the sidewalls of the microchannel and plasma was collected from the collection outlet. The throughput capability to process blood cells is 7,600 cells $\mathrm{s}^{-1}$, which is higher than the conventional hydrophoretic techniques. The throughput of hydrophoretic sorter that was used to enrich WBCs from RBCs is 4,000 cells $\mathrm{s}^{-1}{ }^{20}$.Fig. $6 \mathrm{~b}$ and $\mathrm{c}$ show the blood cells before isolation (b) and after isolation (c). After DEP-active hydrophoretic isolation, the plasma reached a purity of up to $94.2 \pm 3.6 \%(n=3)$ from diluted whole blood. The yield of plasma extracted from diluted whole blood was $15.6 \pm$ $2.5 \%(n=3)$.

Isolating plasma is clinically important because it may contain viruses and their biomarkers ${ }^{2}$. The blood cells (platelets, Red blood cells, and WBCs) should be removed in advance otherwise plasma containing contaminants may affect the final analytical result. The DEP-active hydrophoretic device is a tunable microfluidic system that may become a powerful tool to separate plasma from whole blood. It could potentially be a critical component in advanced lab on a chip bioanalysis devices.

\section{Conclusions}

A DEP-active hydrophoretic device has been developed in this study. The device has been demonstrated to separate micro particles with different sizes and to extract plasma from diluted whole blood. The lateral positions of particles can be controlled by varying external electric field. Not only did the large cells (red blood cells and WBCs) form a hydrophoretic ordering, the small cells (platelets) focused simultaneously in our microfluidic device. The high throughput was achieved by increasing the height of the channel (up to $90 \mu \mathrm{m}$ ) compared with the conventional hydrophoretic channel with the channel height less than twofold particle diameter. The size limit, i.e., the smallest diameter to be focused, was enlarged by exerting negative DEP force on particles in the $z$-axis generated by interdigitised electrodes. After filtration, $99.4 \%$ of $3 \mu \mathrm{m}$ beads and $99.8 \%$ of 10 $\mu \mathrm{m}$ beads were removed from initial particle suspending. This technique offers a new method for filtrating blood cells from whole blood and extracting plasma with a purity of $94.2 \%$ and a yield of $16.5 \%$ in a high throughput manner. Moreover, this work solved the bottle-neck issue of low throughput of DEP based devices. The DEP-active hydrophoresis also provides a tunable microfluidic system that could potentially be used in advanced lab on a chip bioanalysis devices.

\section{Acknowledgments}

This work is supported by the University of Wollongong -China Scholarship Council joint scholarships and the CSIRO Office of Chief Executive Top-up Scholarship. Special thanks go to Dr. Ronald Sluyter from University of Wollongong for providing the blood samples. The authors also thank Assistant Professor Sung
Young Choi, Kyung Hee University, Korea, for helpful discussions. This work was performed in part at the Melbourne Centre for Nanofabrication (MCN) in the Victorian Node of the Australian National Fabrication Facility (ANFF)

\section{Notes and references}

${ }^{a}$ School of Mechanical, Materials and Mechatronic Engineering University of Wollongong, Wollongong, NSW 2522, Australia. E-mail: weihuali@uow.edu.au

${ }^{b}$ School of Electric, Computer and Telecommunication Engineering, University of Wollongong, Wollongong, NSW 2522, Australia

${ }^{c}$ CSIRO Materials Science and Engineering, Private Bag 33, Clayton, VIC, 3169, Australia. E-mail: yonggang.zhu@csiro.au

${ }^{d}$ Melbourne Centre for Nanofabrication/Australian National Fabrication Facility, 151 Wellington Road, Clayton, VIC, 3168, Australia

1. J. A. Davis, D. W. Inglis, K. J. Morton, D. A. Lawrence, L. R. Huang, S. Y. Chou, J. C. Sturm and R. H. Austin, Proc. Natl. Acad. Sci. U. S. A., 2006, 103, 14779-14784.

2. M. Kersaudy-Kerhoas and E. Sollier, Lab Chip, 2013, 13, 3323-3346.

3. S. Choi, T. Ku, S. Song, C. Choi and J.-K. Park, Lab Chip, 2011, 11, 413-418.

4. M. Kersaudy-Kerhoas, Microfluid. Nanofluid., 2010, 8, 105-114.

5. T. Tachi, Anal. Chem., 2009, 81, 3194-3198.

6. S. Thorslund, Biomed. Microdevices, 2006, 8, 73-79.

7. J. Moorthy, Lab Chip, 2003, 3, 62.

8. D. S. Lee, Y. H. Choi, Y. D. Han, H. C. Yoon, S. Shoji and M. Y. Jung, ETRI Journal, 2012, 34, 226-234.

9. L. R. Huang, E. C. Cox, R. H. Austin and J. C. Sturm, Science, 2004, 304, 987-990.

10. S. Yang, Lab Chip, 2006, 6, 871.

11. D. Di Carlo, Lab Chip, 2009, 9, 3038-3046.

12. J. Zhang, W. Li, M. Li, G. Alici and N. T. Nguyen, Microfluid. Nanofluid., 2013, 1-12.

13. A. J. Mach and D. Di Carlo, Biotechnol. Bioeng., 2010, 107, 302311.

14. S. Choi, S. Song, C. Choi and J.-K. Park, Anal. Chem., 2009, 81, 5055.

15. S. Choi, S. Song, C. Choi and J. K. Park, Small, 2008, 4, 634-641.

16. S. Choi and J.-K. Park, Lab Chip, 2007, 7, 890-897.

17. S. Choi and J.-K. Park, Lab Chip, 2009, 9, 1962-1965.

18. S. Song and S. Choi, J. Chromatogr. A, 2013, 1302, 191-196.

19. S. Choi and J. K. Park, Anal. Chem., 2008, 80, 3035-3039.

20. S. Choi, S. Song, C. Choi and J.-K. Park, Lab Chip, 2007, 7, 15321538.

21. J. Shi, X. Mao, D. Ahmed, A. Colletti and T. J. Huang, Lab Chip, 2008, 8, 221-223.

22. C. Liu, T. Stakenborg, S. Peeters and L. Lagae, J. Appl. Phys., 2009, 105 .

23. Y. Ai, B. Mauroy, A. Sharma and S. Qian, Electrophoresis, 2011, 32, 2282-2291.

24. C. H. Yu, J. Vykoukal, D. M. Vykoukal, J. A. Schwartz, L. Shi and P. R. C. Gascoyne, J. Microelectromech. S., 2005, 14, 480-487.

25. D. Holmes, H. Morgan and N. G. Green, Biosens. Bioelectron., 2006, 21, 1621-1630.

26. I. F. Cheng, H.-C. Chang, D. Hou and H.-C. Chang, Biomicrofluid., 2007, 1 . 
27. N. Demierre, T. Braschler, R. Muller and P. Renaud, Sensor. Actuat. B: Chem., 2008, 132, 388-396.

28. E. B. Cummings and A. K. Singh, Anal. Chem., 2003, 75, 4724-4731.

29. P. K. Thwar, J. J. Linderman and M. A. Burns, Electrophoresis, 2007, 28, 4572-4581.

30. J. Zhu and X. Xuan, Electrophoresis, 2009, 30, 2668-2675.

31. J. Zhu, T.-R. J. Tzeng, G. Hu and X. Xuan, Microfluid. Nanofluid., 2009, 7, 751-756.

32. C. Church, J. Zhu, J. Nieto, G. Keten, E. Ibarra and X. Xuan, J. Micromech.Microeng., 2010, 20.

33. J. Zhu and X. Xuan, J. Colloid Interface Sci., 2009, 340, 285-290.

34. M. Li, S. Li, W. Cao, W. Li and W. Wen, J. Micromech.Microeng., 2012, 22, 095001.

35. A. Lenshof and T. Laurell, Chem. Soc. Rev., 2010, 39, 1203-1217.

36. Y. Nakashima, S. Hata and T. Yasuda, Sens. Actuators, B, 2010, 145, 561-569.

37. S. Yan, W. Li, J. Zhang, M. Li, G. Alici, H. Du and R. Sluyter, Sci. Rep., submitted.

38. S. Choi, Anal. Chem., 2009, 81, 50-55.

39. J. Zhang, M. Li, W. H. Li and G. Alici, J. Micromech.Microeng., 2013, 23.

40. M. Li, S. Li, J. Wu, W. Wen, W. Li and G. Alici, Microfluid. Nanofluid., 2012, 12, 751-760.

41. T. B. Jones, Electromechanics of Particles, Cambridge Unviersity Press, Cambridge, 1995.

42. L. Landau and E. Lifshitz, Fluid mechanics, 1987.

43. M. Zborowski, G. R. Ostera, L. R. Moore, S. Milliron, J. J. Chalmers and A. N. Schechter, Biophys. J., 2003, 84, 2638-2645.
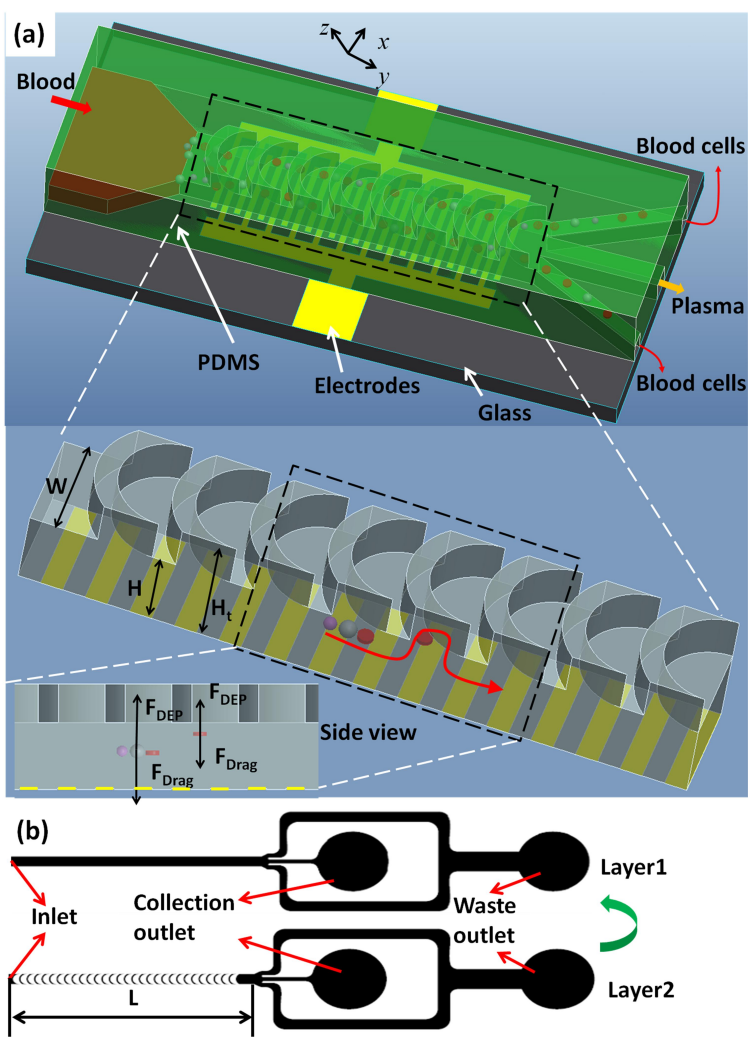

Fig. 1 Isolation of plasma in a DEP-active hydrophoretic device. (a) Overview of microfluidic device showing the isolation of plasma. The insets shows large cells (Red blood cells and WBCs) and small cells (platelets) can be focused onto the sidewall by a steric hindrance mechanism under a certain external electric field, and forces exerted on the cells on different cross sections. (b) Schematic illustrating two-step photolithography. Layer 2 with 30 grooves was aligned and placed on top of layer 1. Blood plasma is collected from the collection outlet. 

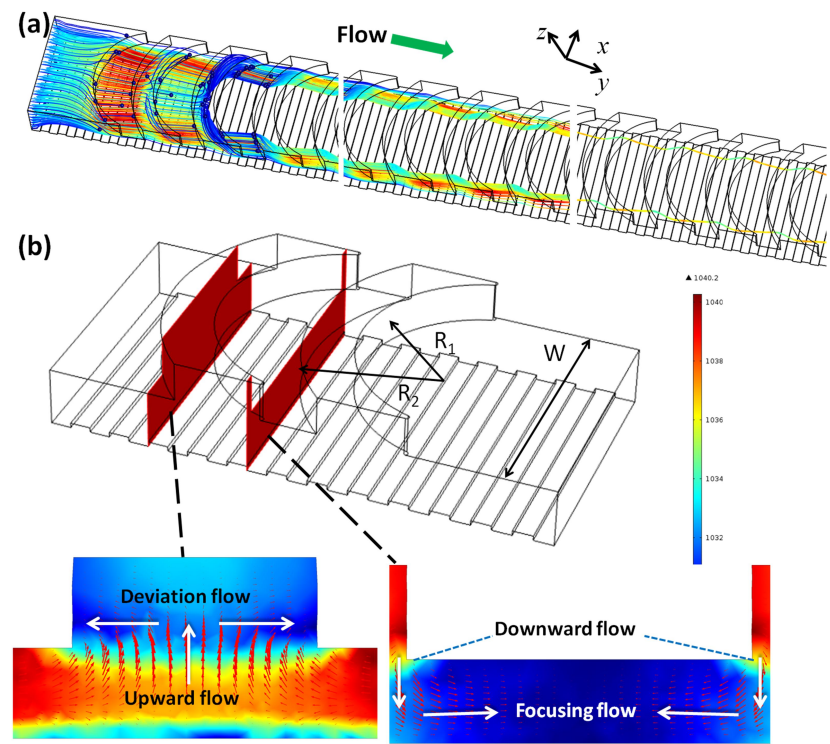

Fig. 2 (a) Simulated particle trajectories in three sections of the mcirochannel. Left image shows the entrance section of the microchannel, middle image the middle section and right the exit section. The steric effect makes the cells diffuse out of convective vortices and assume hydrophoretic ordering. The color of trajectories denotes particle velocity. The red represents high speed, while blue represents low speed. (b) The schematic diagram of the micro-channel and the simulated results of flow field and pressure in the cross-sections. The flow direction is along the $y$ axis. The anisotropic microstructure induces helical recirculation, composing of an upward, deviation, downward, and focusing flow. The red arrows in the insets are velocity vectors. The background color of the insets denotes pressure field. The scale bar refers to the value of pressure. 

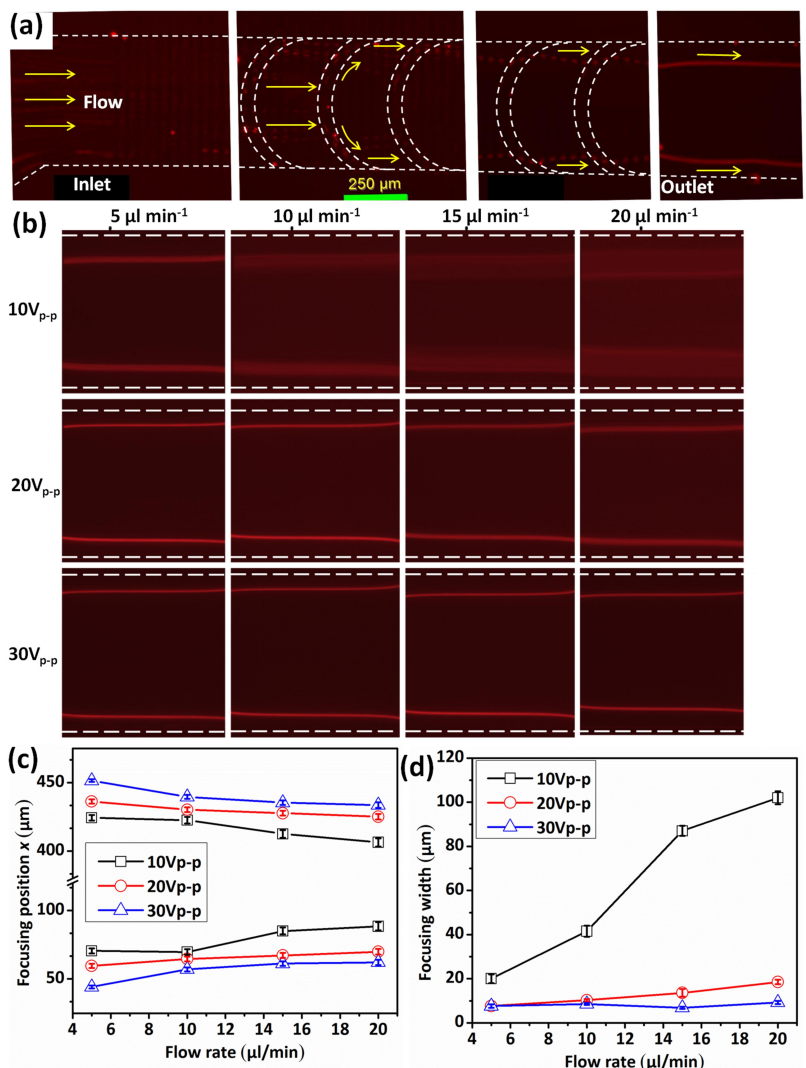

Fig. 3 Focusing patterns of $3-\mu \mathrm{m}$ particles. The applied voltage was changing from 10 to $20 \mathrm{Vp}$-p. The applied flow rate ranged from 5 to 20 $\mu \mathrm{L} \mathrm{min}{ }^{-1}$. The particles had a uniform distribution at the inlet. Optical microscopy images showing the trajectories of particles along the whole channel (a) and the focusing patterns of particles at the outlet (b). (c) Measured focused positions and (d) focused widths from the fluorescent profiles. The average value of 3 times the measurement. 

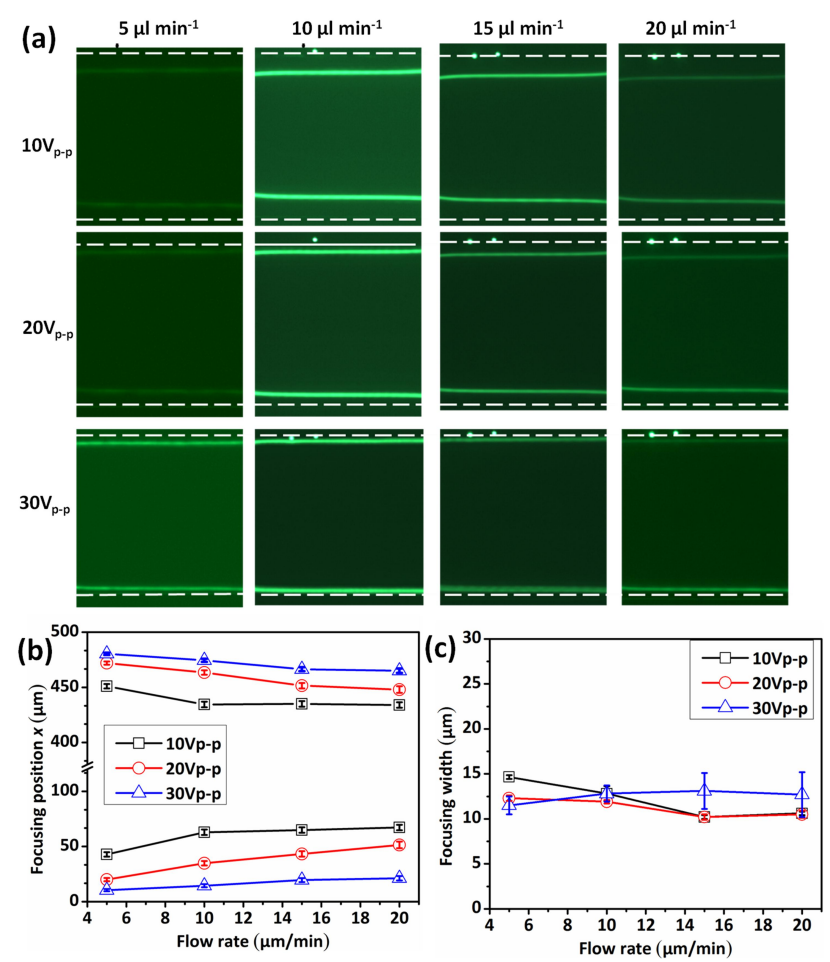

Fig. 4 Focusing patterns of $10-\mu \mathrm{m}$ particles. The applied voltage was changing from 10 to $20 \mathrm{Vp}$-p. The applied flow rate ranged from 5 to 20 $\mu \mathrm{L} \mathrm{min}^{-1}$. The particles had a uniform distribution at the inlet. (a) Optical microscopy images showing the focusing patterns of particles in the microfluidic channel. (b) Measured focused positions and (c) focused widths from the fluorescent profiles. The average value of 3 times the measurement. 

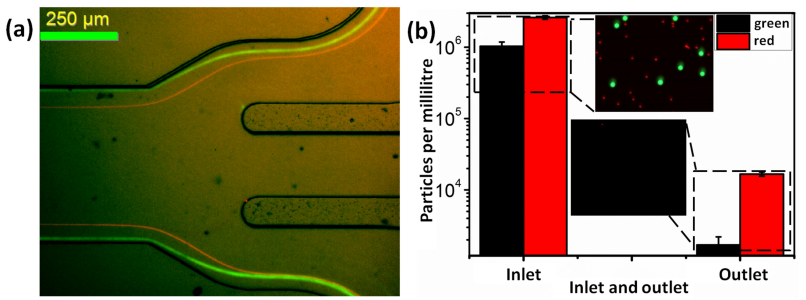

Fig. 5 Filtration of $3 \mu \mathrm{m}$ and $10 \mu \mathrm{m}$ beads. The applied flow rate was 15 $\mu 1 \mathrm{~min}^{-1}$ and external voltage was $20 \mathrm{Vp}$-p. The beads were evenly injected into the micro-channel. (a) Optical microscopy images showing the trajectories of $3 \mu \mathrm{m}$ and $10 \mu \mathrm{m}$ particles. (b) Particle concentration measured from both inlet and collection outlet. Insets illustrate the particles before and after filtration. The average value of 3 times the measurement. 

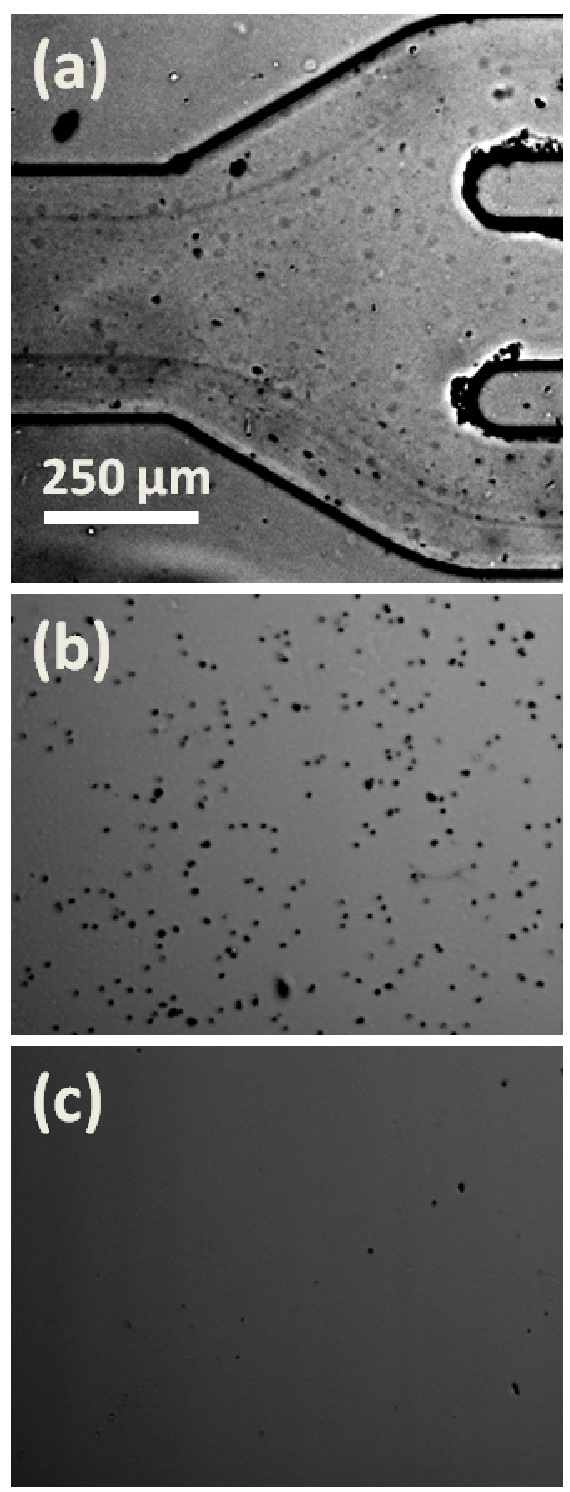

Fig. 6 Isolation of plasma from diluted whole blood. The applied flow rate was $10 \mu \mathrm{min}^{-1}$ and external voltage was $20 \mathrm{Vp}$-p. The blood cells were evenly injected into the micro-channel. (a) Optical micrographs showing the trajectories of blood cells. Blood cells (b) before isolation and (c) after isolation are shown in bright-field images. These images were acquired with $40 \times$ magnification. 